\title{
Progress in Bioactivities of Phlorotannins from Sargassumi
}

\author{
Jiaoyang Li, ${ }^{a}$ Yuxin Wang, ${ }^{a}$ Ruihua Guo, ${ }^{a, b, c}$ Bin Bao, ${ }^{a, b, c}$ and Wenhui $\mathrm{Wu}^{*, a, b, c}$ \\ ${ }^{a}$ College of Food Science and Technology, Shanghai Ocean University, Shanghai 201306, China \\ ${ }^{b}$ Shanghai Engineering Research Center of Aquatic-Product Processing \& Preservation, \\ Shanghai 201306, China \\ ${ }^{c}$ National Experimental Teaching Demonstration Center for Food Science and Engineering, \\ Shanghai Ocean University, Shanghai 201306, China
}

Email:whwu@shou.edu.cn

\begin{abstract}
Bioactive components of marine species have received great attention in recent years, especially phlorotannins isolated from brown algae. This paper summarized progress in the structures and bioactivities of phlorotannins including antibacterial, antioxidant, anticoagulant, anti-diabetic, anti-hypertensive, inhibiting HIV, anti-tumor, anti-allergic, anti-inflammatory, defense activity, and deodorant activities.
\end{abstract}

Keywords Sargassum, phlorotannins, bioactivities

\section{Introduction}

The sea is an enormous resource with rare compounds due to various marine species with a half of the total global biodiversity. As a source of rare bioactive substances, marine organisms grow rapidly. ${ }^{[1]}$ Marine algae is a rich source of various bioactive compounds. According to the composition of pigments, marine macro algae or seaweed is classified into Chorophyta (green algae), Phaeophyta (brown algae) and Rhodophyta (red algae). ${ }^{[2]}$ Brown algae contains a higher level of polyphenol in comparison to red and green algaes. ${ }^{[3]}$ The active ingredients in brown algae is a variety of phloroglucinolbased polyphenols such as phlorotannins.

Sargassum is a part of the family Phaeophyta Fucales Sargassaceae. It is generally more than $1 \mathrm{~m}$ high, grows mostly on rocks with a depth of 2 to $3 \mathrm{~m}$ in a low tide or moss. There are about 250 kinds of Sargassum in the world. Chinese local Sargassum includes more than 130 kinds of seaweed, accounting for approximately $50 \%$ of the world's total number of species of Sargassum. It is widely distributed in Xisha Islands, Nansha Islands, Hainan Island, Nao Island and Wenzhou. ${ }^{[4]}$ Sargassum has been regarded as an important source of bioactive natural substances recently; moreover, many metabolites isolated from marine algae displays bioactivities. Consequently, it is focused on isolation of rare bioactive constituents from edible seaweed.

Residents in Guangdong, Hainan and other places in China utilize various Sargassum as "seaweed" medicinal materials instead, such as Sargassum honeri, Sargassum siliquastrum, Sargassum thunbergii, Sargassum milutum, Sargassum muticum, Sargassum hemiphyllum, Sargassum wachellianum and Sargassum henslowianum. In addition to China, Sargassum is also a traditional medicine in Japan and South Korea. Soamsan, a herbal remedy of traditional Korean medicine, has long been prescribed to treat cardiovascular diseases in elderly patients. ${ }^{[5]}$

Herein, we summarized the structures and bioactivities of phlorotannins including antioxidant properties, antibacterial activity, anti-diabetic effects, anticoagulant activity, anti-carcinogenic activity, anti-allergic effects, anti-inflammatory activity, anti-HIV-1 activity, anti-hypertensive effects, anti-hyperlipidemia effects and deodorant.

\section{Structures of phlorotannins}

Since the 1990s, pre-acetylation, column chromatography, ${ }^{1} \mathrm{H}$ NMR, ${ }^{13} \mathrm{C}$ NMR, and MS have been employed to isolate phlorotannins from brown algae and identify hundreds of new phlorotannins. Phlorotannins are tannin derivatives consisting of several phloroglucinol units linked with each other in different ways. $^{[6-9]}$

\section{Fucols}

Fucols's linked way is intermolecular ring of pyrogallol linked to $\mathrm{C}-\mathrm{C}$ bond. Fucols distributes mainly in Fucus and Dictyomles. $X x$. Fucol is involved in difucol (1), trifucol (2), tetrafucol A (3) and tetrafucol B (4), ${ }^{[10,11]}$ as shown in Figure 1.

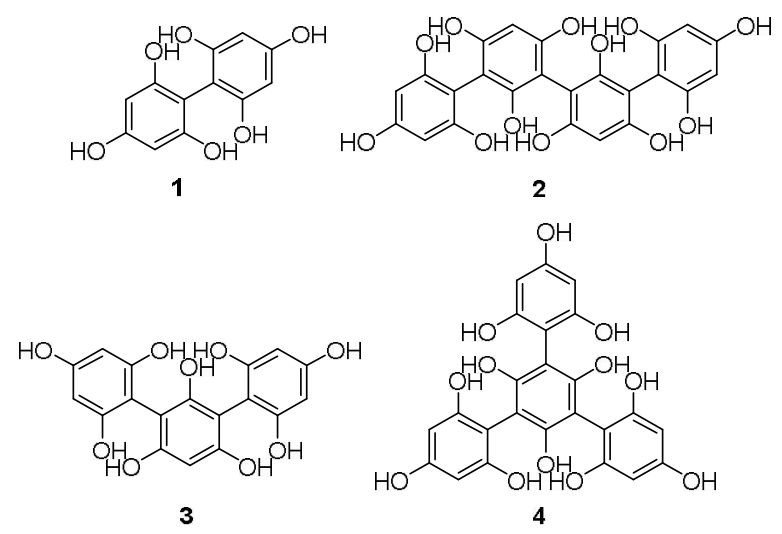

Figure 1 The structures of compounds 1-4. 


\section{Phlorthols}

Pyrogallol is linked by a diaryl ether bond in phlorthols, which distributes mainly in Cystoseira and okamurae. Heo et al. ${ }^{[12]}$ isolated diphlorethohydroxycarmalol from I. okamurae including triphlorthol $C(5)$, triphlorthol $A(6)$, diphlorthol (7), triphlorthol B (8), tetraphlorthol B (9), tetraphlorthol C (10), tetraphlorthol $A(11) .{ }^{[10]}$ Their structures are shown in Figure 2.

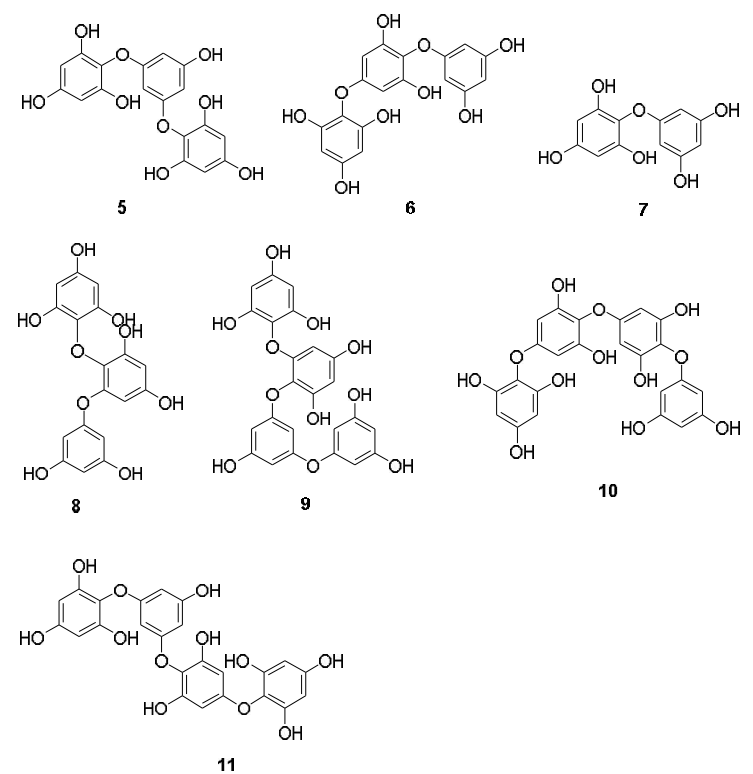

Figure 2 The structures of compounds 5-11.

\section{Fucophlorethols}

Pyrogallol is intermolecularly linked to the ring $\mathrm{C}-\mathrm{C}$ bond and ether linkage in fucophlorethol. Fucophlorethol distributes mainly in Fucus, Sargassum and spinuligerum. Yoon et al. ${ }^{[13]}$ isolated fucodiphloroethol $G$ from Eisenia stolonifera. Fucophlorethol includes fueodiphlorethol $C$ (12), fueotetraphlorethol D (13), fueodiphlorethol B (14), fueotriphlorethol $F$ (15), and fueotetraphlorethol $\mathrm{E}(16),{ }^{[10]}$ whose structures are shown in Figure 3.
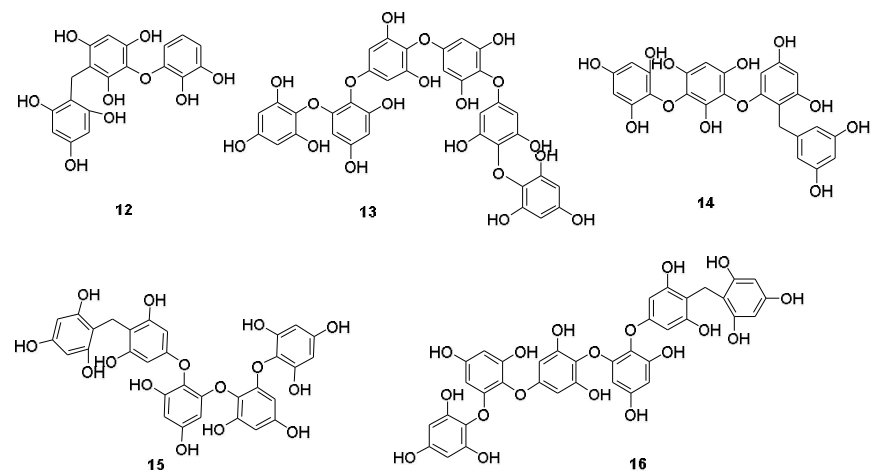

Figure 3 The structures of compounds 12-16.

\section{Fuhalols}

Phloroglucinol is linked by an ether bond of an aryl group in fuhalol, but its monomer contains an additional hydroxyl group to form an ortho. Fuhalol distributes in Sargassum and spinuligerum. Five different structures of fucophlorethols, including bifuhalol $B(17)$, tetrafuhalol $B(18)$, tetrafuhalol $C$ (19), hexafuhalol $B(20)$ and bifuhalol $B(21),{ }^{[10]}$ are shown in Figure 4
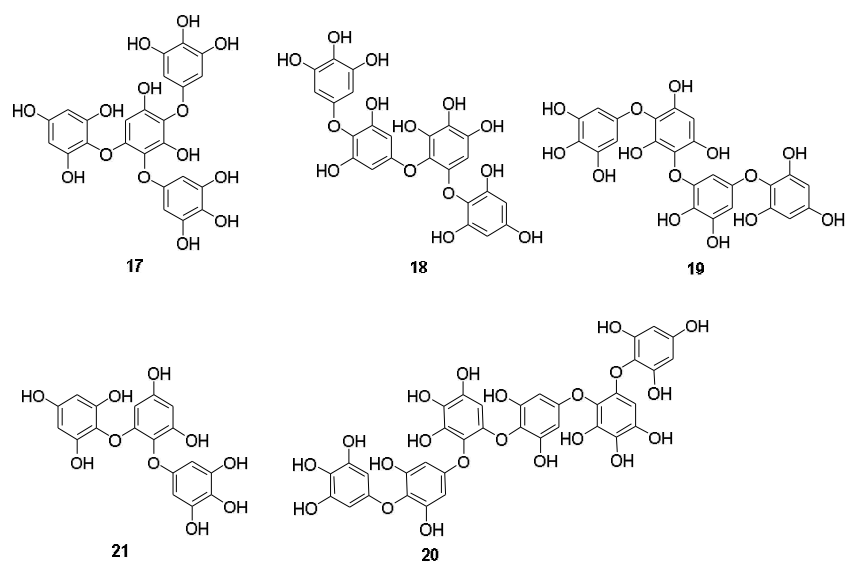

Figure 4 The structures of compounds 17-21.

\section{Eckols}

Eckols's linked way is that two phloroglucin units are cyclized to form diphenyl dioxygen in the dehydrogenated oligomers of three pyrogallol units. Eckols distributes mainly in Sargassum and Eisenia stolonifera. Joe et al. ${ }^{[14]}$ isolated Eckols and dieckol from Eisenia stolonifera. Shibata et al. ${ }^{[15]}$ isolated Eckols from Eisenia bicyclis, E. Eisenia cava and Eisenia kurome. Two different structures of Eckols, Fucofuroeckol A (22) and Fucofuroeckol C (23), ${ }^{[10,16]}$ are shown in Figure 5.<smiles>Oc1cc(O)cc(Oc2c(O)cc(O)c3c2Oc2c(cc4oc5c(O)c(O)cc(O)c5c4c2O)O3)c1</smiles>

22

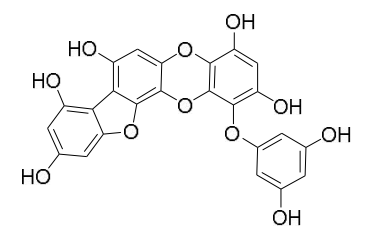

Figure 5 The structures of compounds 22 and 23 .

\section{Halogenated phlorotannins}

I,2,3,5-Tetrahydroxybenzene is a unit of polyphenol in halogenated phlorotannin, which is present in fucoidan in the form of 1,2,3,5-tetrahydroxybenzene-2,5-dithiophosphate or 1,2,3,5-tetrahy droxybenzene-2-sulfuric acid ester (24, Figure 6). ${ }^{[16]}$ Halogenated phlorotannins distributes in Fucus and Dictyomles.<smiles>Oc1cc(CCc2cc(Br)c(O)c(Br)c2)cc(O)c1O</smiles>

Figure 6 The structure of compound 24.

\section{Active phlorotannins}

Phlorotannins used widely in biomedical industry possess strong activities containing eckol (25), ${ }^{[12]}$ phlorofucofuroeckol-B (26), ${ }^{[17]}$ phlorofucofuroeckol-A (27), ${ }^{[18]}$ dioxynodehydroeckol (28), ${ }^{[19]}$ 2-phloroeckol (29), dieckol (30), 8,8'-bieckol (31), ${ }^{[20]}$ and 6,6 '-bieckol $(32),{ }^{[21]}$ as shown in Figure 7. 

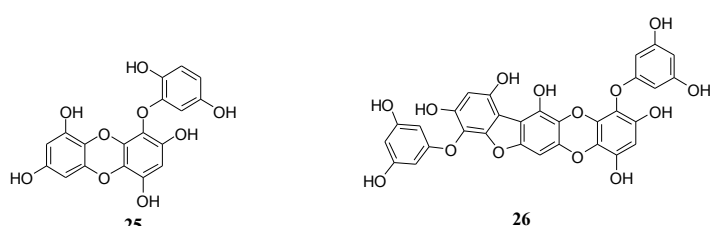

25

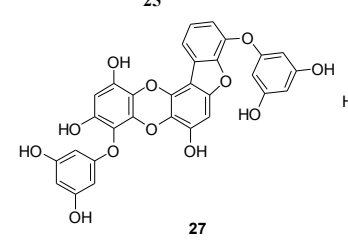

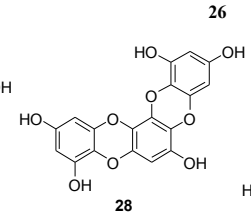

$\mathrm{OH}$

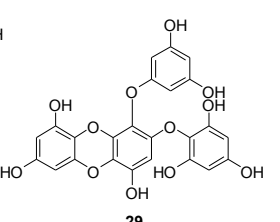<smiles>OC1=CC(O)=C2Oc3c(O)c(O)cc(O)c3OC1=C(O)c1oc3c(Oc4cc(O)cc(O)c4)c(O)cc(O)c3c(O)c(c(O)cc1O)O2</smiles><smiles></smiles>

30

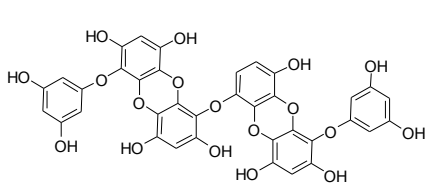

32

Figure 7 The structure of compound 25-32.

\section{Bioactivities and potential health benefits of phlorotannins}

\section{Antibacterial activity}

Lin et al. ${ }^{[22]}$ determined the inhibition of various bacteria using the flat-panel method from phlorotannins dialysate of Sargassum thunbergii Kuntze and Sargassum kjellmanianum Yendo. The results showed that bacteriostatic effect and the concentration of phlorotannins, relative molecular mass were closely related. The bacteriostatic effect becomes stronger with increase of the concentration. It was found that Sargassum tenerrimum (a kind of Sargassum) had bioactive compounds with antibacterial activity by GC-MS analysis. The phlorotannins with antibacterial activity include fueodiphlorethol C (12), fueotetraphlorethol D (13), fucofuroeckol-B (14), eckol (25), phlorofucofuroeckol-A (27), dioxinodehydroeckol (28), 8,8'-bieckol (31) and dieckol (30), etc. ${ }^{[23]}$ Dieckol and 8,8'-bieckol (32) isolated from Eisenia kurome were successfully inhibited the growth of Campylobacter jejuni and Vibrio parahaemolyticus. When minimum inhibitory concentration (MIC) value is $125-250 \mu \mathrm{g} / \mathrm{mL}$, eckol (25) from Eisenia cava indicated potent antimicrobial activity against methicillinresistant Sargassum aureus (MRSA). ${ }^{[24,25]}$ Hudson $^{[26]}$ had studied antiviral (HSV and SINV) compounds from 13 Korean seaweed (Codium fragile, Enteromorpha linza, Ulva pertusa, Colpomenia bullosa, Ishige okamurai, Sargassum sagamianum, Scytosiphon Iomentaria, Undaria pinnatifida, Carpopeltis affinis, Corallina pilulifera, Grateloupia turuturu, Symphyocladia latiuscula, Symphyocladia marchantioides) extracts and found different antiviral compounds in the seaweed extract.

\section{Antioxidant activity}

Heo et al. ${ }^{[12]}$ found that several phlorotannins purified from Ecklonia cava displayed antioxidant activity and protected cells against hydrogen peroxide-induced damage. ${ }^{[27,28]}$ The phlorotannins had significant radical scavenging activities against the superoxide anion (50\% effective concentration value: $6.5-8.4 \mu \mathrm{M})$ and diphenyl picryl hydrazide(DPPH) $(50 \%$ effective concentration value: $12-26 \mu \mathrm{M}$ ), and they were more effective in comparison to ascorbic acid and $\alpha$-tocopherol. ${ }^{[29]}$ The phlorotannins with antioxidant activity include eckol (25), phlorofucofuroeckol A (27), dieckol (30), and 8,8-bieckol (33). Moreover, it showed potent inhibitory activity against phospholipid peroxidation in a liposome system under its concentration of $1 \mu \mathrm{M}^{[30]}$ In general, phenolic hydroxyl dissociation is an important antioxidant mechanism of phlorotannins. The reduction of phenolic hydroxyl is one of the commonalities of phenolic compounds. The phenolic hydroxyl groups in the alginate polyphenols of phloroglucinol can be used as hydrogen donors and they are easily oxidized by oxygen through free radicals in the air. Currently, recognization of biological antioxidants refers mainly to the free radical scavenging, inhibition of lipid peroxidation of the active substance. The phlorotannins were purified from brown algae Sargassum kjellmanianu and their inhibitory effect on the rancidity of fish oil was also studied. It was found that its antioxidant efficiency was 2.6 times higher than that of butylated hydroxytoluene (BHT). ${ }^{[31]}$ Therefore, phlorotannins derived from marine brown algae have strong antioxidant activities against free radical mediated oxidation damage.

\section{Anticoagulant activity}

Anticoagulant is a class of substance that can delay blood coagulation or accelerate fibrinolysis; moreover, it is also an important drug for prevention and treatment of thrombotic diseases. ${ }^{[32]}$ The phlorotannins purified from sargssum thunbeergii Kuntze indicated anticoagulant activities such as coagulation time $(C T)$, bleeding time(BT), activated partial thromboplastin time (APTT), prothrombin time (PT), thrombin time (TT). This nature has been examined by animal experiments. Moreover, the anticoagulant effect depends on its molecular weights. The higher the molecular weight is, the stronger the anticoagulant activity is. The results indicated that the group whose molecular weight is bigger than $1.0 \times 10^{5}$ owns the best anticoagulant activity in comparison to other groups, meaning that the anticoagulant activity is that phlorotannins can inhibit effectively the platelet aggregation and lower the content of $\mathrm{TXB}_{2}$ while the content of 6-Keto-PGF in plasma increases. In addition, it can also inhibit significantly the concentration of free calcium in platelet. ${ }^{[33]}$

\section{Anti-diabetic activity}

Diabetes is a genetic or organ disease that leads to insulin hyposecretion. When body lacks insulin, the level of glucose in blood will elevate without control, which results in damage of blood vessels or nervous system. ${ }^{[34]}$ Great efforts are being considered to explore and development potential diabetes drugs from marine organism. A lot of novel and known phloroglucinols have been isolated from brown algae with excellent anti-diabetic properties. Phlorotannin derivatives extracted with Eisenia cava from brown algae can control rats with streptozotocin-induced type I diabetes. A significant phlorotannin isolated from a kind of a brown algae was named Ishige okamurae. The phlorotannin is diphlorethohydroxycarmalol (DPHC), which can delay dietary carbohydrate absorption in intestine, causing the increasing rate of blood glucose level after a meal can be weaken. This is a strong evidence to prove phlorotannin as a potential drugs to treat diabetes. ${ }^{[35]}$ 


\section{Anti-hypertensive activity}

Researchers have focused on the brown algae polyphenols in the study of hypertension recently. Jung et al. ${ }^{[36]}$ observed that the phlorotannins hydrolyzed by flavor protease $E$. cava had a potential ACE inhibitory effect, and the phlorotannins obtained by hydrolyzing the other six kinds of brown algae were combined with synthetic ACE inhibitors such as captopril phase. The phlorotanins had a better antihypertensive effect than captopril phase. The underlying antihypertensive mechanisms of Sargassum include influencing renin-angio-tensin-system and related factor of vascular endothelial cells, inhibiting certain genes invoked in inflammation, agitating $M$ cholinergic receptor, endothelium-independent relaxation. Artan et al. ${ }^{[37]}$ reviews the current progress on antihypertensive components and the underlying mechanisms of Sargassum, the purpose of which is to deepen the relevant study of Sargassum.

\section{Inhibition of HIV activity}

The inhibition of HIV activity of phlorotannins is mainly based on inhibiting the development of cancer through some mechanisms such as clearance of free radicals, inhibition of tumor gene expression, inhibition of cell proliferation, inhibition of metabolic detoxification and metabolic activation, physiological and immunological regulation. ${ }^{[38]}$ CD4 is the primary cellular receptor for HIV entry. Chinese used traditional Chinese medicine to treat HIV and developed new drugs by optimizing CD4-based HIV inhibitors. ${ }^{[39]}$

\section{Antitumor activity}

The anti-tumor activity of phlorotannins is mainly based on inhibition of the occurrence of cancer through several mechanisms such as clearance of free radicals, inhibition of tumor gene expression, inhibition of cell proliferation, inhibition of metabolic detoxification and metabolic activation, and physiological and immunological regulation. ${ }^{[40]} \mathrm{Yu}^{[41]}$ reported that the phlorotannin compounds of $S$. argentea possessed strong anti-tumor activity and inhibited the proliferation of human lung adenocarcinoma A-549 cells and human hepatoma BEL-7402 cells. High molecular weight fucoidan has anti-tumor effects than low molecular weight phlorotannins. ${ }^{[42]}$ determined the antitumor activity of kelp phlorotannins in vitro by the thiazolyl blue colorimetry. When the crude extract of kelp phlorotannins reached $6.91 \mu \mathrm{g} / \mathrm{mL}$, the three kinds of tumors could kill HeLa, MCF-7 and PC-12 cell lines cell.

\section{Anti-allergic activity}

Atopic dermatitis (AD) as a pruritic skin disease is hardly cured. Patients also suffered from asthma, food allergy and allergic rhinitis. The elevation of serum immunoglobulin $E(\lg E)$ levels and eosinophilia is the main cause of AD's symptom. Enormous antibody is used to remove the $\lg E$ from $A D$ patients for controling $A D$, so the therapy is very expensive. ${ }^{[3,44]}$ Ryu et al. ${ }^{[45]}$ disclosed that phlorotannins isolated from the edible marine brown alga could prevent histamine's release from anti-DNP IgE-sensitizedrat basophile leukemia cells. Basophils and most cells have the acceptor, which can combine with Ig $\mathrm{E}$ the trigger allergic reactions. Phlorotannins derivatives can be developed to candidate drug to prevent allergic due to histamine.

\section{Anti-inflammatory activity}

Inflammation is the complex biological response of vascular tissue to harmful stimulation, and it is a protective measure to remove harmful stimulate. However, this self-defense also brings side effects on body. Researchers investigated that the side effect can be alleviated by phlorotannins. ${ }^{[46]}$ Some phlorotannins isolated from marine brown algae (eckol, dieckol, etc.) have effective influence on common diseases associated with inflammation such as arthritis, atherosclerosis and cancer. A new polyphenol derived from the liang-bulido phlorofucofuroeckol A and phlorofucofuroeckol B possesses the same activity in comparison to existing anti-inflammatory agent such as catechinic acid and gallic acid. In vitro experiments of the rat basophils (RBL)-2H3, the phlorofucofuroeckol-B showed a good anti-inflammatory effect. ${ }^{[47]}$

\section{Other activities}

In addition to the main activity of phlorotannins, phlorotannins have defense and deodorant activities. Chemical defensive activity is mainly reflected in the radiation, heavy metal chelation and other aspects. ${ }^{[48]}$ Yoon et al. ${ }^{[13]}$ found that three kinds of brown algae showed strong deodorization activity toward methanethiol. The deodorizing activity of polyphenolic compounds in natural wood was stronger than that of natural deodorant such as chlorophyll and copper chlorophyll.

\section{Conclusion}

The phlorotannin compound is a secondary metabolite of brown algae and it is a natural marine bioactive substance with a variety of activities. Through extraction, isolation and purification of phlorotannins, phlorotannins with different purity have been obtained to meet the market demand for different products in recent years. However, some restrictive factors such as complex composition of phlorotannins, site of action, mechanism of action, inadequate structural identification, affect its efficacy in-depth research. Chinese Sargassum is rich in resources, and Sargassum is rich in polyphenols. The important direction of future research is to improve the structure, bioactivities and use value of polyphenols.

\section{Acknowledgement}

The work was supported by the National Natural Science Foundation of China (No. 81502955), the Doctoral Scientific Research Foundation of Shanghai Ocean University (No. A2030214300077), the Young Teachers Training Program of Shanghai (No. A12056160002), the Plan of Innovation Action in Shanghai (No. 14431906000), and the Project Funded by Jiangsu Key Laboratory of Marine Pharmaceutical Compound Screening.

\section{References}

[1] Li, Y. X.; Wijesekara, I.; Li Y. Process Biochem. 2011, 46, 2219.

[2] Nwosu, F.; Morris, J.; Lund, V. A.; Stewart, D.; Ross, H. A.; McDougall, G. J. Food Chem. 2011, 126, 1006.

[3] Zubia, M.; Robledo, D.; Freile-Pelegrin, Y. J. Appl. Phycol. 2007 , 19, 454.

[4] Rrms, R. J. Phycol. 2006, 45, 181

[5] Yoon, S. C.; Kim, J. K.; Kwak, D. H. J. Ethnopharmacol. 2004 93, 403.

[6] Li, Y. X.; Wijesekara, I.; Li Y. Process Biochem. 2011, 46, 2221.

[7] Heffernan, N.; Brunton, N. P.; FitzGerald, R. J.; Smyth, T. Mar. Drugs 2015, 13, 522.

[8] Smit, A. J. J. Appl. Phycol. 2004, 16, 247.

[9] Thomas, N. V.; Kim, S. K.; Thomas, N. V.; Kim, S-K. Environ. Toxicol. Pharm. 2011, 32, 327

[10] Yu, S. G. Ph.D. Thesis, Study on Extraction, Purification and Biological Activity of Brown Algae Polyphenols. Qindao University, Qindao, China, 2003, p. 13. 
[11] Artan, M.; Li, Y.; Karadeniz, F.; Lee, S. H.; Kim, M. M.; Kim, S. K. Bioorg. Med. Chem. Lett. 2008, 16, 7921.

[12] Heo, S. J.; Ko, S. C.; Cha, S. H.; Kang, D. H.; Park, H. S.; Choi, Y. U.; Kim, D.; Jung, W. K.; Jeon, Y. J. Toxicol. In Vitro 2009, 23, 1123.

[13] Yoon, N. Y.; Chung, H. Y.; Kim, H. R.; Choi, J. S. J. Fisheries Sci. 2008, 74, 200.

[14] Joe, M. J.; Kim, S. N.; Choi, H. Y.; Shin, W. S.; Park, G. M.; Kang, D. W.; Kim,Y. K. J. Biol. Pharm. Bull. 2006, 29, 1735.

[15] Shibata, T.; Ishimaru, K.; Kawaguchi, S.; Yoshikawa, H.; Hama, Y. J. Appl. Phycol. 2008, 20, 705.

[16] Yang, H. C.; Dong, S. Y.; Liu, Z. Y.; Guo, Y. H.; Li, R. X.; Zeng, M. Y. J. Chin. Mar. Drugs 2007, 26, 201.

[17] Jung, H. A.; Yoon, N. Y.; Woo, M. H.; Choi, J. S. Fisheries Sci. 2008, 74, 1363.

[18] Sugiura, Y.; Matsuda, K.; Yamada, Y.; Nishikawa, M.; Shioya, K.; Katsuzaki, H.; Imai, K.; Amano, H. Biosci. Biotechnol. Biochem. 2006, 70, 2807.

[19] Kong, C. S.; Kim, J. A.; Yoon, N. Y.; Kim, S. K. Food Chem. Toxicol. 2009, 47, 1653.

[20] Artan, M.; Li, Y.; Karadeniz, F.; Lee, S. H.; Kim, M. M.; Kim, S. K. Bioorg. Med. Chem. Lett. 2008, 16, 7921.

[21] Le, Q. T.; Li, Y.; Qian, Z. J.; Kim, M. M.; Kim, S. K. Process Biochem. 2009, 44, 168.

[22] Lin, C. Ph.D. Physiological Activity of Polyphenols in Two Brown Algae, Qindao University, Qindao, China, 2005, p. 32.

[23] Kobayashi, I.; Muraoka, H.; Hasegawa, M.; Saika, T.; Nishida, M.; Kawamura, M.; Ando, R. J. Antimicrob. Chemoth. 2002, 50, 130.

[24] Eom, S.; Kim, Y. Fish Sci. Technol. 2008, 11, 4.

[25] Braden, K.W.; Blanton J.; Allen, V. G.; Pond, K. R.; Miller, M. F. J. Food Protect 2004, 67, 1826.

[26] Hudson, J.; Leem, K. J. Appl. Phycol. 1999, 11, 429.

[27] Heo, S. J.; Park, P. J.; Park, E. J.; Kim, S. K.; Jeon, Y. J. Eur. Food Res. Technol. 2005, 221, 45. Kang, H. S.; Chung, H. Y.; Jung, J. H.; Son, B. W.; Choi, J. S. Chem. Pharm. Bull. 2003, 51, 1013.

[28] Choi, W. J.; Lee, H. W.; Kim, H. O.; Chinn, M.; Gao, Z. G.; Patel,
A.; Jacobson, K. A.; Moon, H. R.; Jung, Y. H.; Jeong, L. S. Bioorg. Med. Chem. 2009, 17, 1965.

[29] Shibata, T. I. K.; Kawaguchi, S.; Yoshikawa, H.; Hama, Y. J. Appl. Phycol. 2008, 20, 708.

[30] Kahl, R.; Kappus, H. Unters. Forsch. 1993, 196, 335.

[31] Zhi, Z.; Li, X.; Liu, H. Fujian Fisheries 2013, 6, 481.

[32] Jase, B. Food Chem. Toxicol. 2011, 7, 329.

[33] Zimmet, P. A. K.; Shaw, J. Nature 2001, 414, 785.

[34] Heo, S. J.; Park, P. J.; Park, E. J.; Kim, S. K.; Jeon, Y. J. Eur. Food Res. Technol. 2009, 615, 254.

[35] Athukorala, Y.; Jeon, Y. J. Food Sci. Nutr. 2005, 10, 136.

[36] Jung, H. A.; Hyun, S. K.; Kim, H. R.; Choi, J. S. Fisheries Sci. 2006, 72, 1298.

[37] Artan, M. L. Y.; Karadeniz, F.; Lee, S.; Kim, M.; Kim, S. Bioorg. Med. Chem. 2008, 16, 7922.

[38] Guo, H. J.; Li, Y. Z.; Elena E.; Paskaleva, K.; Manoj A.; Jeffrey S.; Chin. Herbal Med. 2014, 6, 267.

[39] Nagai, T.; Yukimoto, T. Food Chem. 2003, 81, 328.

[40] Yu, X. W.; Song, X. Chin. Herbal Med. 2008, 39, 94.

[41] Bai, L. J. Dalian Univ. Technol. 2008, 24, 219.

[42] Sugiura, Y.; Matsuda, K.; Yamada, Y.; Nishikawa, M.; Shioya, K.; Katsuzaki, H.; Imai, K.; Amano, H. Biosci. Biotechnol. Biochem. 2006, 70, 2808.

[43] Sugiura, Y.; Yamada, Y.; Nishikawa, M.; Shioya, K.; Katsuzaki, H. Food Sci. Technol. Res. 2007, 14, 56.

[44] Shim, S. Y.; Le, Q. T.; Lee, S. H.; Kim, S. K. Food Chem. Toxicol. 2009, 47, 557.

[45] Ryu, B.; Li, Y.; Qian, Z. J.; Kim, M. M.; Kim, S. K. Chem. Biol. Interact. 2009, 179, 187.

[46] Shibata, T.; Fujimoto, K.; Nagayama, K.; Yamaguchi, K. J. Food Sci. Technol. 2002, 37, 706.

[47] Kim, Y.; An, R.; Yoon, N.; Nam, T.; Choi, J. Arch. Pharm. Res. 2005, 28, 1378.

Received February 18, 2018 Accepted March 23, 2018 